\title{
Effectiveness of pharmaceutical care at discharge in the emergency department: study protocol of a randomized controlled trial
}

\author{
Regina Kuhmmer ${ }^{1,2^{*}}$, Karine Margarites Lima ${ }^{1,3}$, Rodrigo Antonini Ribeiro ${ }^{1,3}$, Luciano Serpa Hammes ${ }^{1}$, \\ Gisele Alsina Nader Bastos ${ }^{1,4}$, Maria Claudia Schardosim Cotta de Souza 1,3, Carisi Anne Polanczyk 1,2,3, \\ Guilherme Alcides Flores Soares Rollin', Suhelen Caon ${ }^{1}$, Cátia Moreira Guterres ${ }^{1}$, Leni Everson Araújo Leite ${ }^{1}$, \\ Tássia Scholante Delabary ${ }^{1}$ and Maicon Falavigna ${ }^{1,3,5}$
}

\begin{abstract}
Background: Patient education on pharmacological therapy may increase medication adherence and decrease hospitalizations. Our aim is to evaluate the effectiveness of pharmaceutical care at emergency department discharge in patients with hypertension and/or diabetes.

Methods/design: This is a randomized controlled trial. Participants will be recruited from a public emergency department at Restinga district in Porto Alegre, southern Brazil. A total of 380 patients will be randomly assigned into 2 groups at the moment of emergency department discharge after receiving medical orientations: an intervention group, consisting of a structured individual counseling session by a pharmacist in addition to written orientations, or a control group, consisting only of written information about the disease. Outcomes will be assessed in an ambulatory visit 2 months after the randomization. The primary outcome is the proportion of patients with high medication adherence assessed using the Morisky-Green Test and the Brief Medication Questionnaire. The secondary outcomes are reduction of blood pressure, glycated hemoglobin, fasting plasma glucose, quality of life and number of visits to the emergency department.
\end{abstract}

Discussion: Pharmaceutical care interventions have shown to be feasible and effective in increasing medication adherence in both hospital outpatient and community pharmacy settings. However, there have been no previous assessments of the effectiveness of pharmacy care interventions initiated in patients discharged from emergency departments. Our hypothesis is that pharmaceutical counseling is also effective in this population.

Trial registration: ClinicalTrials.gov registration number: NCT01978925 (11 November 2013) and Brazilian Registry of Clinical Trials U1111-1149-8922 (5 November 2013).

Keywords: Pharmaceutical care, Medication adherence, Blood pressure, Diabetes, Emergency

\footnotetext{
* Correspondence: regina.kuhmmer@hmv.org.br

'Institute for Education and Research, Hospital Moinhos de Vento, Rua

Ramiro Barcellos 910, Bloco D, Porto Alegre, RS 90035-001, Brazil

${ }^{2}$ Graduate Program in Cardiology and Cardiovascular Science, Universidade

Federal do Rio Grande do Sul, Porto Alegre, Brazil

Full list of author information is available at the end of the article
} 


\section{Background}

Cardiovascular disease (CVD) is the leading cause of mortality and burden of disease as measured in disability-adjusted life years (DALYs). Globally, high blood pressure and high blood glucose alone are responsible for $13 \%$ and $6 \%$ of deaths, respectively [1].

Treatment of chronic diseases commonly includes the long-term use of pharmacotherapy. Despite the efficacy of these treatments, inadequate medication adherence has been reported to approximately $50 \%$ of patients. Low adherence reduces the effectiveness of treatment, resulting in suboptimal illness control. As a consequence, an increased use of healthcare resources and reduction in patients' quality of life has been observed [2-5]. About 20\% of hospitalized patients experience adverse events after discharge. However, it is estimated that $60 \%$ of these events could be prevented with the proper use of medications [6-9].

Interventions focused on patient education related to pharmacological therapy may increase medication adherence and decrease morbidity [10]. Several interventions for improving adherence have been tested in clinical studies, including motivational and behavior strategies, simplification of dosing regimens, unit dose packaging, educational counseling, refill reminders and self-monitoring [11-16]. A systematic review of studies assessing interventions to improve medication adherence has shown an overall increase of 4 to $11 \%$; however, no single strategy appeared to be superior [9].

Pharmaceutical care interventions for improving medication adherence seem to be feasible and effective, both in hospital and in community pharmacy settings $[17,18]$. Clinical pharmacists are able to identify and intervene to prevent potential problems with prescriptions written prior to discharge from the hospital, and studies have suggested that discharge counseling is able to improve medication adherence [19]. However, there is no evidence in the literature regarding the effectiveness of pharmaceutical care interventions initiated in patients discharged from emergency departaments (ED). In addition, few studies have assessed the impact of these interventions in developing countries, and the effect of these interventions in populations with lower income and educational level is uncertain.

The aim of this study is to compare pharmaceutical care at discharge from the ED with usual care in the adherence to medication prescriptions and in the control of hypertension and/or diabetes.

\section{Methods/design}

\section{Study design}

This is a randomized controlled, single-center study, with blinding of outcome assessors. A pilot study with ten patients was previously conducted in order to test study logistics and data collection instruments. Participants will be recruited from a public ED at Restinga district in Porto Alegre, southern Brazil. Porto Alegre is the 10th largest city in Brazil, with 1.5 million inhabitants. Restinga is a low-income district of Porto Alegre, with approximately 100,000 people and a Human Development Index (HDI) of 0.700 to 0.799 , whereas Porto Alegre HDI ranges from 0.700 to 0.977 [20]. This ED is the single reference emergency service of the southern region of Porto Alegre, with 8,000 visits monthly. Eligible participants will be randomized to either the intervention or the control group, and will complete a follow-up interview after a period of 2 months (Figure 1).

\section{Inclusion and exclusion criteria}

All patients with ED discharge from 8 am to $6 \mathrm{pm}$, on weekdays, will be screened for eligibility.

\section{Inclusion criteria}

- age $\geq 18$ years;

- previous diagnosis and drug treatment for hypertension and/or diabetes;

- inadequate blood pressure (BP) control (systolic $\mathrm{BP} \geq 160 \mathrm{mmHg}$ or diastolic $\mathrm{BP} \geq 100 \mathrm{mmHg}$ ), inadequate diabetes control (capillary plasma glucose $\geq 200 \mathrm{mg} / \mathrm{dL}$ ) or moderate/severe hypoglycemia (capillary plasma glucose $<70 \mathrm{mg} / \mathrm{dL}$ associated with symptoms or clinical signs).

\section{Exclusion criteria}

- patients without a prescription list or not able to report medications in use;

- patients not residing in the city of Porto Alegre;

- patients unable to answer the questionnaire or to sign the informed consent form;

- patients forwarded to hospitalization.

\section{Study conduct}

Eligible participants will be identified through active screening by a research assistant, reviewing medical notes of admitted patients. The research assistant will describe the project to each potential participant, will provide a straightforward language statement and will invite them to participate in the study. If the participant accepts, written informed consent will be sought before baseline interview and physical examination. All participants will be referred to the study pharmacist for randomization after baseline data collection.

\section{Baseline measures}

Data collection at baseline includes measurement of BP (for patients with hypertension), glycated hemoglobin (HbA1c) and fasting plasma glucose (for patients with diabetes). In 


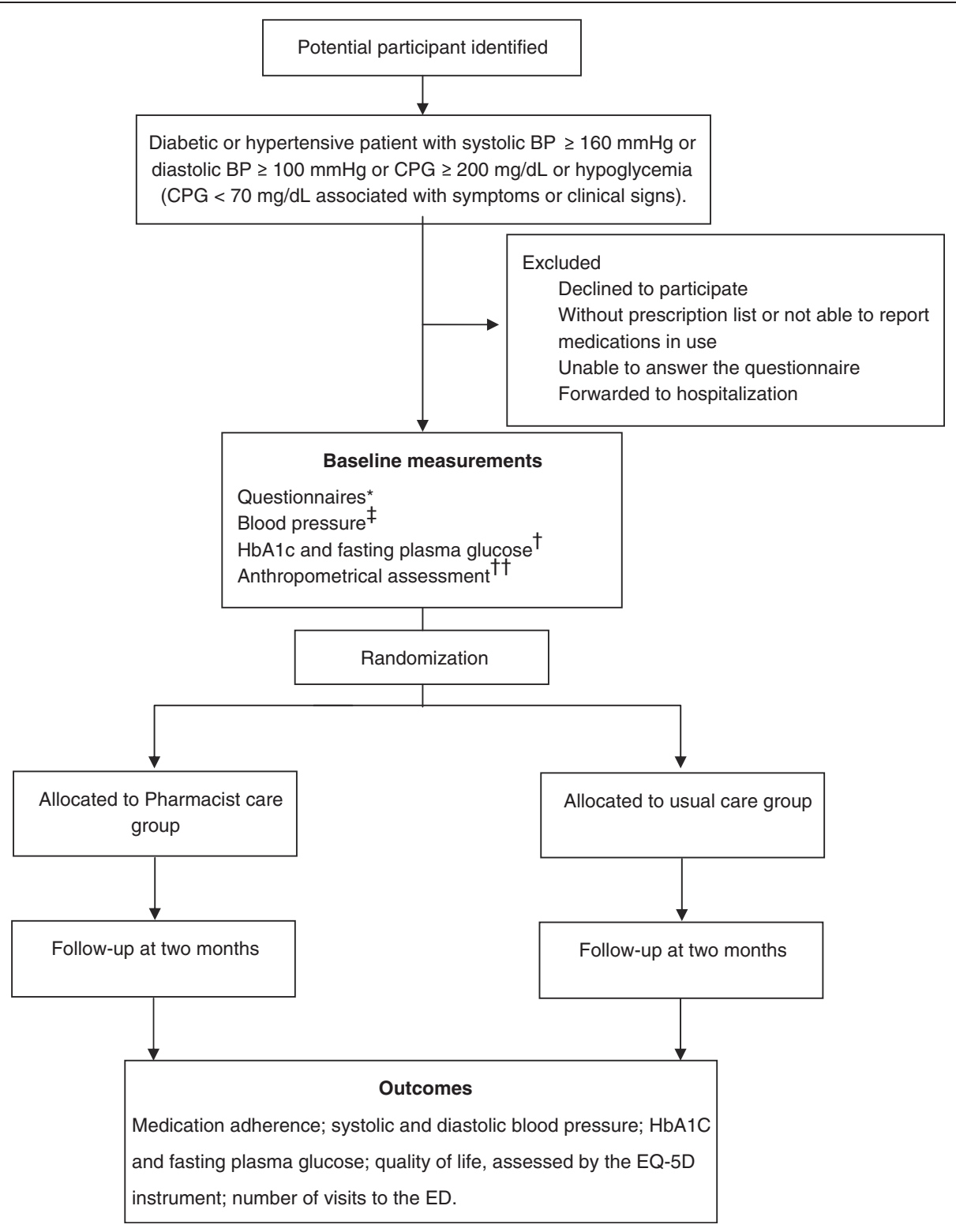

Figure 1 Flow diagram of the study design. *Questionnaires: sociodemographic variables; comorbidities; medication adherence; physical activity; alcohol consumption. ${ }^{*}$ For patients with hypertension. ${ }^{\dagger}$ For patients with diabetes. ${ }^{+\dagger}$ Anthropometrical assessment: weight, height, waist and hip circumferences. $\mathrm{BP}=$ blood pressure; $\mathrm{CPG}=$ capillary plasma glucose; $\mathrm{HbA} 1 \mathrm{C}=$ glycated hemoglobin .

addition, medication in use, socio-demographic, comorbidity and anthropometrical variables (for example, gender, age, ethnicity, marital and smoking status, alcohol consumption, physical activity, circumferences and body mass index) will be recorded. The medical prescription at discharge will also be documented.

\section{Randomization and participants allocation}

Following baseline data collection, eligible participants will be randomized to either the intervention or the control group in a 1:1 ratio, using a telephonic central for allocation concealment. The randomization sequence will be generated by using a computer program in blocks of four, six and eight. Randomization will be stratified according to the number of different medications in use $(<5$ or $\geq 5)$.

\section{Pharmaceutical care group}

In the ED, immediately after discharge, participants randomized to the pharmaceutical care group will receive intervention coordinated by the study pharmacist. The clinical pharmacist will provide a structured 30-minute intervention for enhancing their medication adherence. 
The recommendations include: discussion on hypertension and/or diabetes, risk of complications, prescribed drug therapy, correct use of medications and proper dosage, possible adverse effects, route of administration, schedule of administration and correct storage. The pharmacist will also emphasize the importance of lifestyle modifications. Printed educational material, with information on hypertension and/ or diabetes medications, including suggested lifestyle interventions (for example, reduce salt and sugar intake, practice regular physical activity, smoking cessation, reducing alcohol consumption, monitor stress levels in day-to-day and reduce weight and keep it within the normal range) was prepared to assist in the intervention and will be handed to patients in the end of the session.

\section{Control group}

In addition to counseling provided by a physician and by the nursing staff during their stay in the ED (usual care), patients randomized to the control group will receive the same printed material information on hypertension and/ or diabetes medications and lifestyle interventions in order to keep patients masked.

\section{Follow-up visit}

An ambulatory follow-up visit will occur 2 months after randomization for the assessment of the study's outcomes. We will contact participants by phone prior to the follow-up visit in order to minimize losses; if necessary, home visits will be performed for those without conditions to attend the follow-up visit in the clinic. Data will be collected by a research assistant who is unaware of the randomization status.

\section{Primary endpoint}

The primary outcome is the proportion of patients with high medication adherence at 2 months. Medication adherence will be assessed subjectively using two validated questionnaires in the Portuguese language: the MoriskyGreen Test (MGT) and Brief Medication Questionnaire (BMQ) [21,22]. The MGT assesses both intentional and unintentional nonadherence and comprises four items. Responses for each item are scored one for 'yes' and zero for 'no' and are added together. A total score of zero represents good adherence and a score of one or more represents suboptimal adherence. The BMQ is composed of eleven questions, divided into three domains (regime, beliefs and memory).

\section{Secondary endpoints}

The secondary outcomes are:

- $\quad$ systolic and diastolic BP, for patients with diagnosis of hypertension;

- HbA1c and fasting plasma glucose, for patients with diagnosis of diabetes;
- quality of life, assessed by the the EuroQol fivedimension (EQ-5D) instrument [23,24];

- number of visits to the ED.

\section{Sample size}

The sample size was estimated assuming a percentage of $50 \%$ nonadherence to the medication treatment in the control group [25]. To detect a 15\% absolute increase in the medication adherence in the intervention group, a sample size of 190 participants will be needed in each group, considering an alpha error of 5\% (bilateral), statistical power of $80 \%$ and $10 \%$ of losses to follow-up.

One of the researchers of the study will be responsible for quality control of the collected information. The participants will be randomly selected to answer a simplified questionnaire containing key issues by telephone. This control will be performed in $10 \%$ of the sample.

\section{Data analysis}

The questionnaires will be entered into the database through processing by optical reader, using the Remark Office $\mathrm{OMR}^{\odot}$ Software (version 8.0 Gravic, Inc., Malvern, PA) . All analyses will follow the intention-to-treat (ITT) principle. For the primary outcome, we will initially consider in the analysis patients with complete follow-up. A secondary analysis will be performed with data of all patients initially randomized, considering, as the worst plausible scenario, that $29 \%$ of the losses to follow-up, in both groups, are adherent. The worst plausible scenario was based on a previous observational study, conducted in 2012 in the same region, showing that 34.5\% (95\% confidence intervals (CI), $29 \%$ to $40 \%$ ) of patients with hypertension assisted in basic health units are adherent according to the same instrument [26]. We will not use any imputation method for continuous outcomes, such as BP and blood glucose. For these outcomes, we will include only those participants who attended the followup visit.

Data will be presented as relative risk (RR) and mean difference (MD), with 95\% CI. Categorical variables will be compared using the chi-square test; continuous variables will be compared using the Student $t$-test or the Mann-Whitney $U$-test. The analysis will be conducted in SPSS for Windows (version 19.0, Redmond, WA, USA) and Stata (version 10, College Station, TX, USA); the statistician will be blinded for the randomization status.

\section{Ethics}

This study has been approved by the Institutional Review Board of Hospital Moinhos de Vento of Porto Alegre (approval number 403.658). Written informed consent will be obtained from each participant at the time of enrolment. The trial has also been registered in the Clinical Trial 
Registry NCT01978925 and in the Brazilian Registry of Clinical Trials U1111-1149-8922, and according to the Brazilian Ethics in Human Research Regulations.

\section{Discussion}

We present a study protocol for a randomized controlled trial to investigate the impact of pharmaceutical care in patients with chronic conditions at discharge from an ED. The study will be conducted in a setting with social vulnerability in a developing country. We already knew that at least half of the people in treatment for hypertension and/or diabetes in the same community do not adhere adequately to medications. As consequence, many would develop undesirable outcomes, such as clinical complications and increase in the use of health services resources that could be avoided with adequate adherence to the medical prescription. The moment of the discharge from the ED may constitute an important opportunity for medication reconciliation, reviewing and providing information related to the medications of continuous use $[27,28]$. In this context, we are proposing an intervention focused on pharmaceutical care at emergency discharge in order to enhance adequate medication use. Our hypothesis is that patients receiving pharmaceutical orientation in the intervention group will present better adherence to pharmacological therapy, resulting in better control of $\mathrm{BP}$ and glycemic profile in our study population.

Several studies have shown that pharmaceutical care in the treatment of patients with hypertension and diabetes has been associated with significant reductions in hospitalizations, visits to the ED and increase in medication adherence $[3,9,17-19]$. These studies were conducted mainly in hospital and ambulatory settings in developed countries. To our knowledge, this study is the first designed to evaluate the effectiveness of pharmaceutical care interventions in patients discharged from a public ED. With this randomized controlled trial we expect to provide evidence regarding the effectiveness of pharmaceutical care for chronic conditions at ED discharge. As consequence, these results may be useful for policy -making related to the development and implementation of pharmaceutical care programs in emergency services.

\section{Trial status}

The trial is currently in the recruitment phase.

\section{Abbreviations}

BMQ: Brief Medication Questionnaire; BP: blood pressure; Cl: confidence interval; CVD: cardiovascular disease; DALY: disability-adjusted life year; ED: emergency department; EQ-5D: EuroQol five-dimension instrument; HbA1c: glycated hemoglobin; HDI: Human Development Index; ITT: intention-to-treat; MD: mean difference; MGT: Morisky-Green Test; RA: research assistant; RR: relative risk.

\section{Competing interests}

The authors declare that they have no competing interests.

\section{Authors' contributions}

RAR and GANB conceived the study. RKN, KML, RAR, LSH, GANB, CAP, GAFSR, SC, CMG and MF participated in the study design. LEAL and TSD will include and collect clinical data of the patients. RKN, CMG, MF and SC developed the study intervention. CMG will deliver the proposed intervention. RKN, KML, RAR, MCSCS and MF will be responsible for data management and analysis. RKN and MF are the study coordinators and RAR is the principal investigator. RKN and MF wrote the manuscript. All authors reviewed and approved the final manuscript.

\section{Acknowledgements}

This study is supported by Institutional Development Program of the Brazilian National Health System (PROADI-SUS). Development Project Technical Operation and Management of Health Services in an intramunicipal Region of Porto Alegre, RS - Districts Restinga and extreme south, according signed by the Ministry of Health with the Hospital Moinhos de Vento, through the adjustment term number 05/2011, signed on 30 December 2011.

\section{Author details}

${ }^{1}$ Institute for Education and Research, Hospital Moinhos de Vento, Rua Ramiro Barcellos 910, Bloco D, Porto Alegre, RS 90035-001, Brazil. Graduate Program in Cardiology and Cardiovascular Science, Universidade Federal do Rio Grande do Sul, Porto Alegre, Brazil. ${ }^{3}$ Graduate Program in Epidemiology, Universidade Federal do Rio Grande do Sul, Porto Alegre, Brazil. " Department of Public Health, Universidade Federal de Ciências da Saúde de Porto Alegre, Porto Alegre, Brazil. ${ }^{5}$ Department of Clinical Epidemiology and Biostatistics, McMaster University, Hamilton, Canada.

Received: 14 May 2014 Accepted: 22 January 2015

Published online: 25 February 2015

\section{References}

1. WHO. Global health risks: mortality and burden of disease attributable to selected major risks. Geneva: World Health Organization; 2009. p. 1-170.

2. Melloni C, Alexander KP, Ou FS, LaPointe NM, Roe MT, Newby LK, et al. Predictors of early discontinuation of evidence-based medicine after acute coronary syndrome. Am J Cardiol. 2009;104(2):175-81.

3. Sokol MC, McGuigan KA, Verbrugge RR, Epstein RS. Impact of medication adherence on hospitalization risk and healthcare cost. Med Care. 2005;43(6):521-30

4. Bitton A, Choudhry NK, Matlin OS, Swanton K, Shrank WH. The impact of medication adherence on coronary artery disease costs and outcomes: a systematic review. Am J Med. 2013;126(4):357.e7-357.e27.

5. luga AO, McGuire MJ. Adherence and health care costs. Risk Manag Healthc Policy. 2014;7:35-44.

6. Forster AJ, Clark HD, Menard A, Dupuis N, Chernish R, Chandok N, et al. Adverse events among medical patients after discharge from hospital. CMAJ. 2004;170(3):345-9.

7. Forster AJ, Murff HJ, Peterson JF, Gandhi TK, Bates DW. The incidence and severity of adverse events affecting patients after discharge from the hospital. Ann Intern Med. 2003;138(3):161-7.

8. Pirmohamed M, James S, Meakin S, Green C, Scott AK, Walley TJ, et al. Adverse drug reactions as cause of admission to hospital: prospective analysis of 18820 patients. BMJ. 2004;329(7456):15-9.

9. Peterson AM, Takiya L, Finley R. Meta-analysis of trials of interventions to improve medication adherence. Am J Health Syst Pharm. 2003;60(7):657-65.

10. Christensen M, Lundh A. Medication review in hospitalised patients to reduce morbidity and mortality. Cochrane Database Syst Rev. 2013;2, CD008986.

11. Schroeder K, Fahey T, Ebrahim S. Interventions for improving adherence to treatment in patients with high blood pressure in ambulatory settings. Cochrane Database Syst Rev. 2004;2, CD004804.

12. Skaer TL, Sclar DA, Markowski DJ, Won JK. Effect of value-added utilities on prescription refill compliance and health care expenditures for hypertension. J Hum Hypertens. 1993;7(5):515-8.

13. Márquez-Contreras E, Martell-Claros N, Gil-Guillén V, de la Figuera-Von WM, Casado-Martínez JJ, Martin-de Pablos JL, et al. Efficacy of a home blood 
pressure monitoring programme on therapeutic compliance in hypertension: the EAPACUM-HTA study. J Hypertens. 2006;24(1):169-75.

14. Haynes RB, Yao X, Degani A, Kripalani S, Garg A, McDonald HP. Interventions to enhance medication adherence. Cochrane Database Syst Rev. 2005;4, CD000011

15. Vervloet M, Linn AJ, van Weert JC, de Bakker DH, Bouvy ML, van Dijk L. The effectiveness of interventions using electronic reminders to improve adherence to chronic medication: a systematic review of the literature J Am Med Inform Assoc. 2012;19(5):696-704

16. Gwadry-Sridhar FH, Manias E, Lal L, Salas M, Hughes DA, Ratzki-Leewing A, et al. Impact of interventions on medication adherence and blood pressure control in patients with essential hypertension: a systematic review by the ISPOR medication adherence and persistence special interest group. Value Health. 2013;16(5):863-71.

17. McDonald HP, Garg AX, Haynes RB. Interventions to enhance patient adherence to medication prescriptions: scientific review. JAMA. 2002;288(22):2868-79.

18. Kaboli PJ, Hoth AB, McClimon BJ, Schnipper JL. Clinical pharmacists and inpatient medical care: a systematic review. Arch Intern Med. 2006;166(9):955-64

19. Sarangarm P, London MS, Snowden SS, Dilworth TJ, Koselke LR, Sanchez $\mathrm{CO}$, et al. Impact of pharmacist discharge medication therapy counseling and disease state education: Pharmacist Assisting at Routine Medical Discharge (project PhARMD). Am J Med Qual. 2013;28(4):292-300.

20. Atlas of human development in the metropolitan region of Porto Alegre Porto Alegre: City Hall /Secretariat for Political Coordination and Local Governance. Metroplan; PNUD; Foundation João Pinheiro, 2008, 32. Available in: http://www.pnud.org.br/publicacoes/atlas_portoalegre/ LivroAtlasRMPA.pdf

21. Morisky DE, Green LW, Levine DM. Concurrent and predictive validity of a selfreported measure of medication adherence. Med Care. 1986;24(1):67-74.

22. Ben AJ, Neumann CR, Mengue SS. The brief medication questionnaire and Morisky-Green test to evaluate medication adherence. Rev Saude Publica. 2012;46(2):279-89.

23. Ferreira $\mathrm{PL}$, Ferreira $L N$, Pereira $L N$. Contribution for the validation of the Portuguese version of EQ-5D. Acta Med Port. 2013;26(6):664-75.

24. Group E. EuroQol - a new facility for the measurement of health-related quality of life. Health Policy. 1990;16(3):199-208.

25. World Health Organization. Adherence to long-term therapies: evidence for action. World Health Organization; 2003: 1-194. Available in: http://whqlibdoc.who.int/publications/2003/9241545992.pd

26. Notti RK. Effectiveness of multidisciplinary intervention on blood pressure control in primary health care [PhD thesis]. Federal University of Rio Grande do Sul, Faculty of Medicine; 2014

27. Eisenhower C. Impact of pharmacist-conducted medication reconciliation at discharge on readmissions of elderly patients with COPD. Ann Pharmacother. 2014;48(2):203-8.

28. VanSuch M, Naessens JM, Stroebel RJ, Huddleston JM, Williams AR. Effect of discharge instructions on readmission of hospitalised patients with heart failure: do all of the Joint Commission on Accreditation of Healthcare Organizations heart failure core measures reflect better care? Qual Saf Health Care. 2006;15(6):414-7.

\section{Submit your next manuscript to BioMed Central and take full advantage of:}

- Convenient online submission

- Thorough peer review

- No space constraints or color figure charges

- Immediate publication on acceptance

- Inclusion in PubMed, CAS, Scopus and Google Scholar

- Research which is freely available for redistribution 